\title{
IoT use Prevalence and Acceptance Modelling in the Kingdom of Saudi Arabia
}

\author{
Abdulaziz Albesher ${ }^{1}$, (Member, IEEE) \\ College of Computing and Informatics, Saudi Electronic University, Riyadh, Saudi Arabia
}

\begin{abstract}
This work examines and models the acceptance and application of the Internet of Things (IoT) in the Kingdom of Saudi Arabia. It first explains the tremendous potential and impact of IoT on different areas of governance in smart cities, the challenges and the security threats faced. Next, the significant improvements accompanied by the introduction of advanced countermeasure techniques such as Blockchain technology are discussed. It proposes an acceptance model of IoT in Saudi Arabia based on well-renowned information systems theories, namely Diffusion of Innovations (DOI) theory, Technology Acceptance Model (TAM) and The Unified Theory of Acceptance and Use of Technology (UTAUT). The current study adopted a quantitative approach to test the model. Statistical analysis of the proposed model validates the effectiveness of the proposed work in modelling of IoT use prevalence and acceptance.
\end{abstract}

Keywords: Internet of Things (IoT), Diffusion of Innovation (DOI), Technology Acceptance Model (TAM), Unified Theory of Acceptance and Use of Technology (UTAUT), Saudi Arabia

\section{INTRODUCTION}

With the widespread of mobile communication devices and wireless network coverage at cheaper rates, the emergence of the Internet of Things (IoT) concept added a massive potential for a variety of devices to connect several objects and people [1]. In 1999 Kevin Ashton was the first to proposed IoT as uniquely identifiable things, objects and their virtual representations in an internet-like structure [2]. Nowadays, the full spread of advanced applications (For example, greenhouse monitoring, healthcare sensors, intelligent transportation) allowed the concept of IoT to become popular. Even though the term IoT become particularly popular in recent years, there is no unified understanding or definition to describe what the IoT actually encompasses. The vision for the IoT has extended and evolved dramatically beyond the technologies of RFID. For instance, The International Telecommunication Union (ITU) now defines the IoT as "a global infrastructure for the Information Society, enabling advanced services by interconnecting physical and virtual things based on, existing and evolving, interoperable information and communication technologies" [3]. Simultaneously, several alternative definitions of IoT proposed. Most of the proposed definitions show stress laid on the thingslobjects which become connected in the IoT. A number of these definitions share a center of interest on Internet-related aspects of the IoT, such as network protocols and schemes. Other definitions focus on IoT related challenges, such as search, data storage, retrieving and managing a massive amount of information [4]. IoT solutions experiencing a dramatic growth that extended to approximately cover all areas of everything.

Furthermore, it resulted in having a great diversity in the fields of application for IoT technologies. Among these application fields, the smart industry considered as the most striking pattern. Smart Industry is a segment of industry 4.0 that concentrates on the development of intelligent and advance production system [5]. In the smart cities and smart home, lots of attention drives to security systems and intelligent thermostats [6], whereas smart water, electricity and gas meters are receiving more focus on smart energy applications. In the context of smart health more interest of applications related to chronic diseases control and patients' observation can found, while in smart transport projects like to mobile park ticketing and vehicle tracking gets more attention [7]. Moreover, in the area of smart city solutions similar to realtime managing of streets, monitoring parking space availability and intelligent streets lighting are being addressed [8] — some of these widely used applications summarized in table 1 .

International Telecommunication Union (ITU), in a recent study, indicated that the era of IoT is approaching very fast. European Technology Platform on Smart Systems Integration predicted the development of IoT industry indicated a decade ago that objects would be connected in 2005, would be partly intelligent between 2015 and 2020, and after 2020, objects will be exceptionally clever [9]. Based on these predictions, companies started to invest in producing various IoTbased services and products. In recent years, numerous acquisitions news of IoT-related technology have been making the headlines; this includes the outstanding takeover of Nest by Google for $\$ 3.2$ billion [10] and the subsequent acquisitions of SmartThings by Samsung and of Dropcam by Nest [11]. IoT is a new horizon and business opportunity as it has been increasingly acknowledged by several practitioners and politicians. The current estimate of the IoT market is predicting a growth of worth $\$ 7.1$ trillion by 2020 [12].

Application of IoT systems brings convenience to consumers' life, improves the living quality and reduces the workload. As an example, L'Oreal has recently introduced La Roche-Posay UV Sense which is the first battery-free wearable 


\title{
International Journal of Advanced Research in Computer and Communication Engineering
}

\author{
Vol. 8, Issue 8, September 2019
}

electronic Ultraviolet (UV) Sensor [13], which can sense and communicate the amount of UV radiation the skin exposed. Ever since the concept of IoT proposed, several enterprises have invested heavily in it. The importance of IoT development has been emphasized by government ministries and industry companies. The IoT could comprehensively apply in different fields such as health care, logistics, transportation, smart cities and homes, property protection. It is expected that the market scale of the IoT industry, especially in health care, transportation, and logistics, could grow dramatically by 2020 [14]. Capturing citizens' perceptions can lead to a better understanding of online government transactions and enable the successful delivery of those transactions. User's perception is especially important to understand the lower acceptance rates for what is considered to be a major investment in developing countries [15]. Saudi Arabia is investing a lot in making the life of its citizens better, and this is one of the major focus areas of its Saudi Vision 2030 [16]. In addition, it can lead to demonstrate the extent of acceptance and usage of such advanced technologies in Saudi Arabia. There are several information systems theories used in literature to model the acceptance of IoT in different areas of application and different countries. Some of the most prominent among these theories include Diffusion of Innovations (DOI) theory, Technology Acceptance Model (TAM) and The Unified Theory of Acceptance and Use of Technology (UTAUT).

Some of the recent implementations of such models include the work done by Talebian and Mishra [17] who predicted the adoption of connected autonomous vehicles using DOI. Wong [18] used DOI for the public acceptance of autonomous rail and passenger-vehicle technologies. Hu et al. [19] analyzed and investigated the dynamic interaction between the DOI process and the external environment. Their study involved the adjustment of retail prices and the profit of an online retailer through a simulation model. Park et al. [20] explored the key determinants of user acceptance of IoT technologies in a smart home environment by combining TAM with a set of five user factors. Lekjaroen et al. [21] proposed acceptance using TAM for an IoT Plant watering system using a gardening platform prototype with soil moisture, temperature, water sensor, grow light, and Android application for the elderly. Solangi et al. [22] used UTAUT to evaluate the acceptance and use of IoT in the healthcare area by medical professionals, clinicians and patients in underserved areas of Pakistan. El-Haddadeh et al. [23] used UTAUT to explain the effect of empowerment, perceived usefulness and privacy-related issues on the perceived value of IoT and its continuous use intentions among UK citizens. Chatterjee et al. [24] combined UTAUT and TAM to highlight the factors affecting the implementation of IoT coupled with artificial intelligence in smart cities. In this work, we highlight the major shift towards IoT technologies and its wide and rapid acceptance and adaptation by developed countries. We then formulate a model and analyze the acceptance and prevalence of IoT technologies in Saudi Arabia by utilizing some state-of-the-art information systems theories. The rest of this work will be organized as follows. Section 2 describes in detail different technical and architectural components of IoT along with some discussion on DOI, TAM and UTAUT. Section 3 describes the formulation of our model and describes different devised variables used in this study. Section 4 presents the application of these models and some test results. The conclusion is presented in section 5, along with some future directions.

Table:I Some of the Most Widely Adopted Applications of Iot

\begin{tabular}{|c|c|}
\hline \multicolumn{2}{|l|}{ Transportation } \\
\hline \multirow{2}{*}{$\begin{array}{l}\text { Public } \\
\text { Transportation }\end{array}$} & $\begin{array}{l}\text { Using GPS tracking devices, we can enable real-time monitoring of buses and } \\
\text { give better information on waiting time. }\end{array}$ \\
\hline & $\begin{array}{l}\text { Using swipe-card information, we can analyze peaks in use and provide a better } \\
\text { supply of buses. }\end{array}$ \\
\hline Traffic & $\begin{array}{l}\text { Using GPS tracking devices or presence detection sensors, we can enable real- } \\
\text { time traffic analytics and have a smarter control of traffic lights to prevent traffic } \\
\text { congestion. }\end{array}$ \\
\hline Public bikes & $\begin{array}{l}\text { Using GPS tracking devices, we can analyze where public bikes are most needed } \\
\text { and also balance the availability of bikes at different locations. }\end{array}$ \\
\hline \multicolumn{2}{|l|}{ Economy } \\
\hline Tourism & $\begin{array}{l}\text { Using beacons, we can push notifications on smartphones based on location and } \\
\text { give people additional information about a place in a city or an art object in a } \\
\text { museum. }\end{array}$ \\
\hline Industry & Smart infrastructures. \\
\hline \multicolumn{2}{|l|}{ Health } \\
\hline Assistance to people & Using a single-push button, a person can send an emergency alert. \\
\hline $\begin{array}{l}\text { Automated } \\
\text { medication }\end{array}$ & $\begin{array}{l}\text { Using medical devices, we can automate the supply of medicine for people with } \\
\text { diabetes or high blood pressure and send alerts to their doctor when the device } \\
\text { detects a problem. }\end{array}$ \\
\hline $\begin{array}{l}\text { Maintenance of } \\
\text { medical devices and } \\
\text { failure detection }\end{array}$ & $\begin{array}{l}\text { Using a sensor, a battery-powered medical device could send a notification when } \\
\text { a battery needs to be replaced. }\end{array}$ \\
\hline
\end{tabular}


International Journal of Advanced Research in Computer and Communication Engineering

Vol. 8, Issue 8, September 2019

\begin{tabular}{|c|c|}
\hline \multicolumn{2}{|l|}{ Environment } \\
\hline Water supply & Using sensors, leakage in water pipes can be detected. \\
\hline Pollution & $\begin{array}{l}\text { Using sensors, we can monitor pollution levels and the amount a polluting } \\
\text { particle emitted. }\end{array}$ \\
\hline \multicolumn{2}{|l|}{ Administration } \\
\hline \multirow{2}{*}{$\begin{array}{l}\text { Electronic identity } \\
\text { device }\end{array}$} & $\begin{array}{l}\text { Using magic bands, people can have all their identity information and important } \\
\text { information updated. }\end{array}$ \\
\hline & Using biometrics, personal magic bands can be secured and prevent identity theft. \\
\hline \multicolumn{2}{|l|}{ Security } \\
\hline Drones & Using computer vision, drones can identify security issues and report them. \\
\hline \multirow{2}{*}{ Fire } & $\begin{array}{l}\text { Using smoke detectors, automated notifications can be sent to intervention } \\
\text { centres. }\end{array}$ \\
\hline & $\begin{array}{l}\text { Using sensors in gas pipes, we can identify potential sources of fire and deal with } \\
\text { them before any damage occur. }\end{array}$ \\
\hline \multicolumn{2}{|l|}{ Transportation } \\
\hline \multirow{2}{*}{$\begin{array}{l}\text { Public } \\
\text { Transportation }\end{array}$} & $\begin{array}{l}\text { Using GPS tracking devices, we can enable real-time monitoring of buses and } \\
\text { give better information on waiting time. }\end{array}$ \\
\hline & $\begin{array}{l}\text { Using swipe-card information, we can analyze peaks in use and provide a better } \\
\text { supply of buses. }\end{array}$ \\
\hline Traffic & $\begin{array}{l}\text { Using GPS tracking devices or presence detection sensors, we can enable real- } \\
\text { time traffic analytics and have a smarter control of traffic lights to prevent traffic } \\
\text { congestion. }\end{array}$ \\
\hline Public bikes & $\begin{array}{l}\text { Using GPS tracking devices, we can analyze where public bikes are most needed } \\
\text { and also balance the availability of bikes at different locations. }\end{array}$ \\
\hline \multicolumn{2}{|l|}{ Economy } \\
\hline Tourism & $\begin{array}{l}\text { Using beacons, we can push notifications on smartphones based on location and } \\
\text { give people additional information about a place in a city or an art object in a } \\
\text { museum. }\end{array}$ \\
\hline Industry & Smart infrastructures. \\
\hline \multicolumn{2}{|l|}{ Health } \\
\hline Assistance to people & Using a single-push button, a person can send an emergency alert. \\
\hline $\begin{array}{l}\text { Automated } \\
\text { medication }\end{array}$ & $\begin{array}{l}\text { Using medical devices, we can automate the supply of medicine for people with } \\
\text { diabetes or high blood pressure and send alerts to their doctor when the device } \\
\text { detects a problem. }\end{array}$ \\
\hline $\begin{array}{l}\text { Maintenance of } \\
\text { medical devices and } \\
\text { failure detection }\end{array}$ & $\begin{array}{l}\text { Using a sensor, a battery-powered medical device could send a notification when } \\
\text { a battery needs to be replaced. }\end{array}$ \\
\hline \multicolumn{2}{|l|}{ Environment } \\
\hline Water supply & Using sensors, leakage in water pipes can be detected. \\
\hline Pollution & $\begin{array}{l}\text { Using sensors, we can monitor pollution levels and the amount a polluting } \\
\text { particle emitted. }\end{array}$ \\
\hline \multicolumn{2}{|l|}{ Administration } \\
\hline \multirow{2}{*}{$\begin{array}{l}\text { Electronic identity } \\
\text { device }\end{array}$} & $\begin{array}{l}\text { Using magic bands, people can have all their identity information and important } \\
\text { information updated. }\end{array}$ \\
\hline & Using biometrics, personal magic bands can be secured and prevent identity theft. \\
\hline \multicolumn{2}{|l|}{ Security } \\
\hline Drones & Using computer vision, drones can identify security issues and report them. \\
\hline \multirow{2}{*}{ Fire } & $\begin{array}{l}\text { Using smoke detectors, automated notifications can be sent to intervention } \\
\text { centres. }\end{array}$ \\
\hline & $\begin{array}{l}\text { Using sensors in gas pipes, we can identify potential sources of fire and deal with } \\
\text { them before any damage occur. }\end{array}$ \\
\hline
\end{tabular}

II. IOT AND INFORMATION SYSTEMS MODELING

A. Inside the IoT

The Internet of Things can be defined as "network connecting things" that links several objects and the internet with a network protocol using the information sensor equipment. Common examples of information sensor equipment are radio 


\title{
International Journal of Advanced Research in Computer and Communication Engineering
}

\author{
Vol. 8, Issue 8, September 2019
}

frequency identification technology (RFID) sensors and two-dimensional codes. Thus, it facilitates communication between people and objects, or among objects [7], [19]. Hence, IoT can realize the auto-identification and information sharing of products through the internet by using technologies such as RFID. Usually, the concept of the Internet of Things consists of four major components including applications, heterogeneous access, sensing, information processing and services, besides other components such as privacy and security. IoT use sensor equipment to carry information and connect objects via an internet protocol (for example, radio frequency identification technology and quick response code). In the world of IoT, using technologies such as RFID allows information sharing and auto-identification of items possible in few seconds.

Nowadays, IoT provides companies and governments with numerous competitive advantages. It facilitates companies' operations and helps to demonstrate information beyond the data such as when, how, where and why the data is extracted. Moreover, due to the complexity of this technology, various cybersecurity challenges need to be considered to produce a secure IoT-based system suitable for handling governments and companies' operations. The use of blockchain technology can help to reduce cybersecurity threats demonstrated from using their IoT system. Blockchain technology is one of the confidential, reliable and scalable technologies. It is designed to keep track of different connected devices that offer secure processing of transactions among devices. In addition, blockchain technology relies on decentralized databases, which indicates that the attackers will struggle to expose their operations. It is not a centralized database which makes the system sustainable, reliable and eliminate any single points of failure. For an improved defense against cyberattacks, companies use blockchain to confirm device identity, protect sensory information in transport, and control access to urban automated facilities [25].

\section{B. Acceptance Modelling and Information Systems Theories}

There are several information systems theories adopted in different studies to model the acceptance of new technology in society, region or culture. The most prominent of these are the following.

1) DOI: Diffusion of Innovation (DOI), also named as innovation diffusion theory (IDT), used to study the spread of innovation in society and has been first introduced by Rogers in 1962 [26]. This theory was developed several times until the best model reached by Rogers in 1995. Diffusion of Innovation assumes that individual's adoption of innovation can be categorised into five segments based on individual's innovativeness or based on their willingness while using a new idea: innovators, early adopters, early majority, late majority, and laggards.

Innovation attributes have a significant role in affecting an individual's rate for adopting innovation [26]. These innovation attributes are complexity, relative advantage, observability, compatibility and trialability. Further development of DOI has been made by Moore and Benbasat [27] to provide new robust attributes to measure individuals' perception of innovation in information technology and boost the research in information systems. The proposed attributes are ease of use, compatibility, result demonstrability, visibility, the voluntariness of use, relative advantage, trialability and image.

2) TAM: Technology Acceptance Model (TAM) was introduced by Davis [28] based on the ground of Theory of reasoned action. Perceived ease of use and perceived usefulness are the two main perspectives that lead this model assumed to influence an individual's acceptance towards using the use of a system. Perceived ease of use defined as "referring to the degree to which a person believes that using a particular system would be free from effort". Whereas perceived usefulness (PU) defined as "the degree to which a person believes that using a particular system would enhance his/her job performance" [28].

Technology Acceptance Model is a reliable and powerful model to predict an individual's willingness to adopt a new system in different contexts. This model is well-established and simple to understand the attributes that affect the user's acceptance of technology, besides realizing the drivers that prevented user's acceptance. Furthermore, this model support researchers and practitioners to understand the impact of the exogenous factors on endogenous factors. According to Davis [28], high rates of perceived usefulness and perceived ease of use result in increasing users' intention to use technology.

3) UTAUT: Venkatesh et al. [29] introduced the Unified Theory of Acceptance and Use of Technology to understand the user's willingness to accepting the use of new technology. UTAUT used several models to synthesize and achieve a common perspective of users technology acceptance. Eight models compared, assessed and integrated to introduce the UTAUT model. These models are Theory of Reasoned Action (TRA), Theory of Planned Behaviour (TPB), Technology Acceptance Model (TAM), Diffusion of Innovation (DOI), Motivational Model (MM), combined TAM - TPB (C-TAM-TPB), Model of PC Utilization (MPCU) and Social Cognitive Theory (SCT).

Venkatesh et al. [29] stated that the UTAUT model helps to offer a deep prediction of an individual's behavior, which cannot be determined by using a certain model. All the previous models used in developing UTAUT consist of a number of independent variables. These independent variables contributed to the development of UTAUT variables. Effort expectancy, social influence, facilitating conditions and performance expectancy found as significant variables that have a direct impact on an individual's acceptance and usage [29]. Other moderating variables (e.g. age, gender, experience and voluntariness of use) also found vital in the UTAUT model to understand an individual's behavioral toward using a system. This study aims to understand the extent of acceptance and usage of IoT technology in Saudi Arabia. Such 


\title{
International Journal of Advanced Research in Computer and Communication Engineering
}

\author{
Vol. 8, Issue 8, September 2019
}

advanced technologies add great advantages to different life aspects like economy, transportation and health as presented in table 1. Therefore, relevant researches were reviewed to fulfil this gap and to understand the effective variables. There are a number of variables that could be highly effective in the study of IoT use prevalence when it comes to the country of Saudi Arabia. These include perceived usefulness, age, gender, education level, prior internet experience, perceived intelligence, the intention of use and social influence, to name a few.

\section{CONCEPTUAL MODELING}

This section elaborates the conceptual methodology of the proposed scheme. This study comprises of four phases. The first phase initialized by the literature survey and the proposition of a conceptual model. The second phase starts with the survey development, and different associated data collected along with. It is followed by the analysis and results of data collected on the proposed model to conclude this phase. In the third phase, we concentrate on data collection through interviews. For this purpose, the initial step is of interviews development. After completing the development of the interviews, the interviews were conducted to collect data from different stakeholders and tech-savvies, including academicians, IT professionals, government officials etc. Finally, the analysis and results for this phase are carried out. In the last phase, we revise our model based on the analysis and results in the previous phases by adding, dropping or updating different parameters and variables. This step followed by the conclusion step, which finalizes the developed model, and it is then ready to be tested on the real-world data. In the following subsections, we elaborate on the basic steps discussed herewith and explain their functionality concerning the proposed research. Moreover, only phase one and two will be covered in this research paper due to the limitation of the current study.

\section{A. Sample and procedure}

In line with empirical research in technology acceptance, such as presented in [29], the current study adopted a quantitative approach to test the proposed model. For this purpose, a survey employed due to its many advantages present in literature. The current study focusses on testing hypothesized relationships in the context of technology acceptance. In addition, the proposed variables in this study were developed and validated by the models mentioned above of the user's acceptance and adoption of technology. IBM SPSS Statistics Grad Pack 25.0 was used to analyze the collected data and to conduct many validation tests. Therefore, using the survey as a source to collect data would be suitable from the epistemological, ontological and methodological perspective. The sample is collected using a nonprobabilistic, self-selection method, and should, therefore, be considered a convenience sample. A survey employed in this study due to its advantages. More specifically, the empirical data were collected utilizing a self-administrated questionnaire that contains 54 questions. The target sample for this survey was users who were interested in using IoT systems. Participation was voluntary and no financial incentive offered to take the survey. A total of 302 selfadministered surveys disseminated to the students and a total of 212 surveys were returned, which indicate a response rate of $70.2 \%$. There were 195 valid questionnaires after the exclusion of invalid questionnaires due to duplications or empty fields. Out of the 195 participants, the gender split was 95 males and 100 females, which corresponds to $48.71 \%$ and $51.28 \%$ of the sample size. Their self-rated Web-based learning experience was either some or experienced.

\section{B. Measurement development}

In this work, the questions (items) of the conducted survey was developed based on previous literature review and the aim of this study. This allowed ensuring that quoted items are valid and reliable to measure the proposed variables of the targeted phenomena. The study survey was first pre-tested to avoid any inconsistency of the content. The survey administered by two professors and two professionals who were mainly interested and experienced in the IoT field. This process helps to adjust the proposed items and apply any additional modifications. Reliability and validity of the items were checked in the pilot test that included 40 participants. Further details regarding the results discussed in the proceeding results section.

\section{RESULTS}

This section presents the results of the proposed study on IoT use prevalence and acceptance in Saudi Arabia and presents a thorough discussion of these results. Table 2 presents the correlation response of individual variables against different measurements. A set of measurements and tests include Pearson's Correlation (r), One-tailed significance test (p), and the number of samples $(\mathrm{N})$. We use a number of variables that could be highly effective in the study of IoT use prevalence. These include perceived usefulness, age, gender, education level and prior internet experience. Pearson's correlation represents a linear relationship between two variables, where the values may lie between +1 and -1 . A value of 0 implies no association between the variables, whereas a positive value indicates direct proportionality between the variable, and a negative value indicates inverse proportionality between them. It is to be noted here that for the gender variable, a positive value indicates a tilt towards male whereas a negative value indicated a tilt towards the female response. As can be seen from the table, for most of the variables the Pearson's correlation values are positive which 


\title{
International Journal of Advanced Research in Computer and Communication Engineering
}

\author{
Vol. 8, Issue 8, September 2019
}

shows a positive association between them to understand user's acceptance of IoT. A negative value of Perceived Usefulness against Education level and Internet Usage indicates that more the samples are literate, the more they are considerate of the security threats and the implicit side effects of technology and its usage.

A negative value of Gender against Perceived Usefulness indicates that more females find IoT technology more useful as compared to males. Similarly, a negative value of Gender against Internet Experience indicates that more females are experienced with the use of the internet as compared to the male population. One-tailed significance test indicates the critical area of a distribution to be one-sided so that it could lie either on the positive side or the negative side, but not both. This means that the critical area of distribution can be greater than or less than a certain value, but not both at the same time. In this study, one-tail $\mathrm{P}$ value test used to predict the statistical mean differences of perceived usefulness and the groups of demographic variables. Furthermore, it shows how likely these differences are significant and reliable. The $\mathrm{p}$ values from table 2 indicate that the hypothesis is valid and holds for perceived usefulness of IoT for a different class of variables in the present study.

Table: 2 Correlation Response of Individual Variables against Different Measurements

\begin{tabular}{|c|c|c|c|c|c|c|}
\hline \multicolumn{7}{|l|}{ Correlations } \\
\hline & & $\begin{array}{l}\text { Perceived } \\
\text { Usefulness }\end{array}$ & Age & Gender & $\begin{array}{l}\text { Education } \\
\text { level }\end{array}$ & $\begin{array}{l}\text { Internet } \\
\text { Experience }\end{array}$ \\
\hline \multirow{5}{*}{$\begin{array}{l}\text { Pearson } \\
\text { Correlation (r) }\end{array}$} & $\begin{array}{l}\text { Perceived } \\
\text { Usefulness }\end{array}$ & 1.000 & 0.032 & -0.059 & -0.036 & -0.055 \\
\hline & Age & 0.032 & 1.000 & 0.022 & 0.152 & 0.171 \\
\hline & Gender & -0.059 & 0.022 & 1.000 & 0.093 & -0.102 \\
\hline & $\begin{array}{l}\text { Education } \\
\text { level }\end{array}$ & -0.036 & 0.152 & 0.093 & 1.000 & 0.153 \\
\hline & $\begin{array}{l}\text { Internet } \\
\text { Experience }\end{array}$ & -0.055 & 0.171 & -0.102 & 0.153 & 1.000 \\
\hline \multirow{5}{*}{$\begin{array}{l}\text { Significance (p) } \\
\text { (1-tailed) }\end{array}$} & $\begin{array}{l}\text { Perceived } \\
\text { Usefulness }\end{array}$ & & 0.292 & 0.157 & 0.268 & 0.173 \\
\hline & Age & 0.292 & & 0.353 & 0.005 & 0.002 \\
\hline & Gender & 0.157 & 0.353 & & 0.055 & 0.041 \\
\hline & $\begin{array}{l}\text { Education } \\
\text { level }\end{array}$ & 0.268 & 0.005 & 0.055 & & 0.004 \\
\hline & $\begin{array}{l}\text { Internet } \\
\text { Experience }\end{array}$ & 0.173 & 0.002 & 0.041 & 0.004 & \\
\hline
\end{tabular}

\section{CONCLUSION}

In this work, a proposed model for technology acceptance and application of the Internet of Things (IoT) in the Kingdom of Saudi Arabia was demonstrated. In addition, it discussed the large potential and impact of IoT on different areas of governance in smart cities, the associated challenges and the security threats that can be faced. Then, the study proposed an acceptance model of IoT in Saudi Arabia based on well-renowned information systems theories such as DOI, TAM and UTAUT. Moreover, the current study adopted a quantitative approach to test the model. Statistical analysis and results derived from the proposed model validates the effectiveness of the proposed work in modelling of IoT use prevalence and acceptance in Saudi Arabia.

\section{ACKNOWLEDGEMENT}

The author acknowledges the support provided by Saudi Electronic University (SEU), Saudi Arabia, and its endeavors for research excellence.

\section{REFERENCES}

[1]. T. Qiu, N. Chen, K. Li, M. Atiquzzaman, and W. Zhao, "How can heterogeneous Internet of Things build our future: A survey," IEEE Communications Surveys \& Tutorials, vol. 20, no. 3, pp. 2011-2027, 2018.

[2]. K. Ashton, "That 'internet of things' thing," RFID journal, vol. 22, no. 7, pp. 97-114, 2009

[3]. International Telecommunication Union, "Internet of Things Global Standards Initiative," 20-Jul-2015. [Online]. Available: https://www.itu.int/en/ITU-T/gsi/iot/Pages/default.aspx. [Accessed: 23-Sep-2019].

[4]. L. Atzori, A. Iera, and G. Morabito, “The Internet of Things: A survey,” Computer Networks, vol. 54, no. 15, pp. 2787-2805, Oct. 2010. 


\title{
International Journal of Advanced Research in Computer and Communication Engineering
}

\author{
Vol. 8, Issue 8, September 2019
}

[5]. K. B. Swain, G. Santamanyu, and A. R. Senapati, "Smart industry pollution monitoring and controlling using LabVIEW based IoT," in 2017 third international conference on sensing, signal processing and security (ICSSS), 2017, pp. 74-78.

[6]. Zhang Liang, Zheng Leina, Yang Haodong, and Pan Tiejun, "Research of the key technologies of the smart home based on IOT," Applied Mechanics \& Materials, vol. 713-715, p. 2304, Nov. 2014.

[7]. M. M. Alam, H. Malik, M. I. Khan, T. Pardy, A. Kuusik, and Y. Le Moullec, "A Survey on the Roles of Communication Technologies in IoTBased Personalized Healthcare Applications," IEEE Access, vol. 6, pp. 36611-36631, 2018.

[8]. M. You, "Convergence of IoT, Edge and Cloud Computing for Smart Cities," IEEE Cloud Computing, no. 5, pp. 4-5, 2018.

[9]. K. Mekki, E. Bajic, F. Chaxel, and F. Meyer, “A comparative study of LPWAN technologies for large-scale IoT deployment," ICT express, vol. 5, no. 1, pp. 1-7, 2019.

[10]. K. Zia, A. Muhammad, and D. K. Saini, "Modeling and Assessment of Resource-sharing Efficiency in Social Internet of Things," Dependable IoT for Human and Industry: Modeling, Architecting, Implementation, p. 83, 2018.

[11]. J. Garcia and C. Varol, "SmartThings Event Export using SmartApps," in 2019 7th International Symposium on Digital Forensics and Security (ISDFS), 2019, pp. 1-6.

[12]. F. Wortmann and K. Flüchter, "Internet of things," Business \& Information Systems Engineering, vol. 57, no. 3, pp. 221-224, 2015.

[13]. A. A. Albesher, "IoT in Health-care: Recent Advances in the Development of Smart Cyber-Physical Ubiquitous Environments," IJCSNS, vol. 19, no. 2, p. 181, 2019.

[14]. X. Dong, Y. Chang, Y. Wang, and J. Yan, "Understanding usage of Internet of Things (IOT) systems in China: cognitive experience and affect experience as moderator," Information Technology \& People, vol. 30, no. 1, pp. 117-138, 2017.

[15]. M. Díaz, C. Martín, and B. Rubio, "State-of-the-art, challenges, and open issues in the integration of Internet of things and cloud computing," Journal of Network and Computer Applications, vol. 67, pp. 99-117, May 2016.

[16]. Saudi Vision 2030, "Saudi Vision 2030," 2019. [Online]. Available: https://vision2030.gov.sa/en. [Accessed: 23-Sep-2019].

[17]. A. Talebian and S. Mishra, "Predicting the adoption of connected autonomous vehicles: A new approach based on the theory of diffusion of innovations," Transportation Research Part C, vol. 95, pp. 363-380, Oct. 2018.

[18]. R. W. Wong, "Autonomous Rail Technologies: Diffusion, Risk Perception \& Public Acceptance,” Transportation Law Journal, vol. 45, no. 1, pp. 35-47, Jan. 2018.

[19]. S. Hu, B. Hu, and Y. Cao, "The wider, the better? The interaction between the IoT diffusion and online retailers' decisions," Physica A: Statistical Mechanics and its Applications, vol. 509, pp. 196-209, Nov. 2018.

[20]. E. Park, Y. Cho, J. Han, and S. J. Kwon, "Comprehensive Approaches to User Acceptance of Internet of Things in a Smart Home Environment," IEEE Internet of Things Journal, vol. 4, no. 6, pp. 2342-2350, Dec. 2017.

[21]. K. Lekjaroen, R. Ponganantayotin, A. Charoenrat, S. Funilkul, U. Supasitthimethee, and T. Triyason, "IoT Planting: Watering system using mobile application for the elderly," in 2016 International Computer Science and Engineering Conference (ICSEC), 2016, pp. 1-6.

[22]. Z. A. Solangi, M. S. A. Aziz, and Asadullah, "The study of Internet of Things (IoT)-based healthcare acceptance in Pakistan," in 2017 IEEE 3rd International Conference on Engineering Technologies and Social Sciences (ICETSS), 2017, pp. 1-5.

[23]. R. El-Haddadeh, V. Weerakkody, M. Osmani, D. Thakker, and K. K. Kapoor, "Examining citizens' perceived value of internet of things technologies in facilitating public sector services engagement," Government Information Quarterly, vol. 36, pp. 310-320, Apr. 2019.

[24]. S. Chatterjee, A. K. Kar, and M. P. Gupta, "Success of IoT in Smart Cities of India: An empirical analysis," Government Information Quarterly, vol. 35, no. 3, pp. 349-361, Sep. 2018.

[25]. J. Waldo, “A Hitchhiker's Guide to the Blockchain Universe," Communications of the ACM, vol. 62, no. 3, pp. 38-42, Mar. 2019.

[26]. E. M. Rogers, Diffusion of innovations. New York: Free Press of Glencoe, 1962.

[27]. G. C. Moore and I. Benbasat, "Development of an Instrument to Measure the Perceptions of Adopting an Information Technology Innovation," Information Systems Research, vol. 2, no. 3, pp. 192-222, Sep. 1991.

[28]. F. D. Davis, "Perceived Usefulness, Perceived Ease of Use, and User Acceptance of Information Technology,” MIS Quarterly, vol. 13, no. 3, pp. 319-340, 1989.

[29]. V. Venkatesh, M. G. Morris, G. B. Davis, and F. D. Davis, "User Acceptance of Information Technology: Toward a Unified View," MIS Quarterly, vol. 27, no. 3, pp. 425-478, 2003. 